\title{
Adolescent substance misuse: an update on behaviours and treatments
}

\author{
Paul McArdle \& Bisharda Angom
}

\begin{abstract}
SUMMARY
Substance misuse remains relatively common among adolescents. It can be conceptualised as one manifestation of a broader conduct problem or disinhibitory syndrome that is genetically transmitted. Some substances, notably alcohol, have potential direct effects on brain development. Availability of substances is a necessary condition for use, and recent marketing developments have unleashed a range of new agents. Nevertheless, there is considerable scope for prevention, brief intervention, pharmacological and more elaborate psychosocial treatments.
\end{abstract}

\section{DECLARATION OF INTEREST}

P.M. has received honoraria for speaking at pharmaceutical industry events.

Substance misuse emerged as a relatively common behaviour among Westernised young people towards the end of the 20th century. The trend appeared first in English-speaking countries and, at least in the UK, within a context of deteriorating mental health: national cohort studies reveal 'a substantial increase' in emotional and conduct problems in 16-year-olds over a 20year period (Collishaw 2004, 2010). Whether this link with poor mental health is more than coincidental is unclear. However, the substances used may adversely affect maturing cortical white matter, memory, and educational and psychosocial progress during a critical developmental period (McQueeny 2009; Newbury-Birch 2009). In this way they potentially reduce the life chances and perhaps the lifespan (Impinen 2010) of a substantial minority of young users.

Substance misuse is the term used in the UK to refer to patterns of maladaptive use of substances. DSM-IV refers to substance use disorders, substance abuse, which is broadly equivalent to 'misuse', and substance dependence (American Psychiatric Association 1994). However, some physicians have criticised the current dependence concept, particularly the conflation of 'normal' physiological withdrawal following sustained use of opiates for pain control with the compulsive drug-seeking of addiction. Partly in response, DSM-5 proposes dropping 'dependence' as a concept and combining its symptoms with 'abuse' (American Psychiatric Association 2010). This may be more applicable to young people, among whom, some argue, physiological dependence appears to be uncommon (National Treatment Agency for Substance Misuse 2010).

\section{The epidemiology of the problem}

Much of the available epidemiological data on young people still comes from school surveys. UK school survey data show a reduction in numbers reporting being offered drugs and in the proportion reporting ever having tried them: the latter figure fell from 30\% in 2003 to $22 \%$ in 2009 (Fuller 2010). Nevertheless, among the $22 \%$ of 15 -yearolds reporting any use, $10 \%$ of the boys and $6 \%$ of the girls report use on 'more than 10 occasions', indicative of regular use. Hence, among those reporting any use, at least a third are regular users, many or most of whom 'misuse' and $5 \%$ of the total, about $25 \%$ of users, say that they 'need help or treatment'. Although drinking also appears to have declined over the past decade, $1 \%$ of girls and $2 \%$ of boys drink daily, and of those 15 -year-olds who drink, $31 \%$ of boys and $29 \%$ of girls drink 15 or more units per week. About 5\% smoke cigarettes, drink and use drugs. Further, among pupils excluded or truanting from school, there is a recent trend for increasing substance use. More girls than boys first used drugs 'to forget my problems' (13 v. 9\%) but otherwise their patterns of use are now similar. This 'equality' is a major change that has occurred over a generation and, because of their smaller body size, disproportionately risks the health of females (Newbury-Birch 2009).

School-survey data from the USA also show declines in alcohol drinking and cigarette smoking (Johnston 2009; Lopez 2009). However, from a plateau in 2007, cannabis use has again increased. In their report for the National Institute on Drug
Paul McArdle is a consultant child and adolescent psychiatrist. In addition to working in an in-patient unit, he has for some years run a substance misuse service for young people in partnership with adult addiction services. Under the aegis of the Royal College of Psychiatrists, he currently chairs a group of psychiatrists who treat young substance misusers. Bisharda Angom is a consultant child and adolescent psychiatrist (locum) working for West London Mental Health Trust. She is soon to take up a substantive post in a Tier 4 unit in Colchester, Essex. Correspondence Dr Paul McArdle, Fleming Nuffield Unit, Newcastle upon Tyne NE2 3AE, UK. Email: paul. mcardle@ntw.nhs.uk 
Abuse, Johnstone et al (2009) comment that this followed evidence of reduced perception of risk not (yet) apparent in the UK data - which they term 'generational forgetting'. This is a process whereby a particular generation's knowledge of the widely recognised adverse consequences of a highly prevalent action fades as that generation is replaced. The survey also shows that the perceived risks of LSD, inhalants and ecstasy (MDMA) among participants had declined appreciably.

The 2011 European School Project on Alcohol and other Drugs (ESPAD) is an international schools survey of 15- to 16-year-olds repeated on five occasions since 1995. The project, which now covers 30 European countries and 100000 pupils, offers estimates of current trends in substance use (Hibell 2012). In brief, across the continent, substance use has broadly plateaued since 2007, but rates remain higher than in 1995. Initially apparent mainly in the UK and Ireland, binge use of alcohol and lifetime and regular cannabis use spread from west to east and from north to south across the continent. Nevertheless, the UK still has the third highest and Ireland the sixth highest numbers of young people who admitted being drunk in the previous 30 days. Comparing the genders, with the exception of cigarettes (equality), inhalants (girls have caught up with boys in the most recent survey) and tranquillisers (across the duration of the surveys girls have used more than boys), boys consume more substances than girls. The UK is sixth (France is first) in the ranking for use of cannabis during the previous 30 days.

In 2011, cocaine was second only to cannabis as the most tried drug in Europe (European Monitoring Centre for Drugs and Drugs Addiction 2012), although its use is concentrated in a small number of countries (Table 1).

\section{Novel psychoactive substances}

According to European data, there has been a decline in numbers of injecting drug users

TABLE 1 European-Union countries with lowest and highest prevalences of previousmonth cocaine use among 15- to 34-year-olds

\begin{tabular}{|llc|}
\hline Ranking & Countries & $\begin{array}{c}\text { Population } \\
\text { prevalence, \% }\end{array}$ \\
\hline Low-prevalence countries & Romania & 0 \\
& Greece, Lithuania, Norway, Poland & 0.1 \\
& Czech Republic, Estonia, Hungary & 0.2 \\
\hline High-prevalence countries & UK & 2.1 \\
& Spain & 2.0 \\
& Cyprus & 1.3 \\
& Italy & 1.1 \\
\hline
\end{tabular}

Source: European Monitoring Centre for Drugs and Drug Addiction 2011. presenting to clinical services. However, the European Monitoring Centre for Drugs and Drugs Addiction (2012) now reports new drugs emerging at a rate of almost one a week. These are mainly synthetic cannabinoids ( 23 substances) and synthetic cathinones (8), but they also include phenethylamines such as amfetamine and methamfetamine, and natural cathinones. This situation is challenging governments' capacity to regulate (Advisory Council on the Misuse of Drugs 2011). These inexpensively synthesised, unregulated synthetic compounds can circumvent legal controls and have been commonly available over the counter in 'head-shops' or legally from the internet. The molecular structure of cathinone can be altered to produce various compounds, known as cathinones or cathinone derivatives (Fig. 1), and analysis suggests that most of the new agents seen in the UK are stimulants, similar in effect to amfetamine (Advisory Council on the Misuse of Drugs 2010a, 2011).

Noting legislation that would come into effect in December 2009 to control synthetic cannabinoid receptor agonists such as 'Spice', the UK's Advisory Council on the Misuse of Drugs (ACMD) argued for a similar response to other 'legal highs', focusing on the cathinones, of which mephedrone is the class member most commonly appearing in police drug seizures (Iversen 2009). Owing to their reduced ability to cross the bloodbrain barrier, the cathinones may be less potent than amfetamines but they can cause pronounced autonomic effects, tachycardia, hypertension and vasoconstriction leading to blue peripheries, as well as agitation and depression as the stimulant effect wanes. Interestingly, despite the reported marketing of cathinones as plant fertiliser or bath salts, the ACMD stated that they have no recognised efficacy or suitability for such uses.

Since recent UK control of mephedrone, some websites are said to have switched to selling a structurally related compound, naphyrone, a triple monoamine reuptake inhibitor (dopamine, serotonin and noradrenaline). Drugs such as dexamfetamine, which interact selectively with the dopamine transporter, have psychostimulant properties; those such as MDMA (ecstasy)

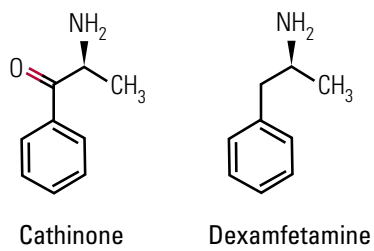


interact selectively with the serotonin transporter and have 'empathogenic' profiles'; and triple uptake inhibitors such as cocaine combine these properties (Advisory Council on the Misuse of Drugs 2010b). Consequently, naphyrone may have a cocaine-like profile, while being more potent than cocaine. Sometimes naphyrone is marketed as NRG-1, but test purchases are said to show a markedly inconsistent range of chemicals. The toxic effects are likely to be similar to those of other stimulants. The ACMD warns that naphthyl compounds have been shown to have carcinogenic properties.

\section{What causes substance misuse?}

\section{Genetic contribution}

Substance misuse is clearly linked with environmental adversities such as family and community breakdown. However, young people who misuse substances commonly exhibit a complex inherited predisposition, of which early use of drugs, alcohol or cigarettes may be a behavioural marker (Costello 2010; Green 2010). For instance, data from the Minnesota Twin Family study (McGue 2008) confirm the long-held view that use of alcohol before the age of 15 is associated with increased risk of later alcohol misuse. Moreover, in a comparison with boys without early alcohol use, early drinking was linked to a range of adverse outcomes: the odds ratio for antisocial personality disorder was 5.8 , for drug dependence 3.2 , nicotine dependence 1.7 (and major depression 1.3). With the exception of a much lower risk for antisocial personality disorder, the pattern of associations was similar for girls. However, the risk of adverse outcomes, including alcohol dependence, associated with early-onset problem behaviour not related to alcohol misuse was sometimes even higher. Compared with young people without early problem behaviour, the risk for lifetime alcohol dependence among early smokers and early drug users was around 7 times higher. The corresponding risk for young people who had been in trouble with the police or had had early sexual intercourse was also considerably higher. McGue et al referred to this array of behaviours as 'disinhibitory psychopathology'.

A population of adopted and non-adopted adolescents (King 2009) revealed that young people living with an alcohol-dependent adoptive parent were more likely to drink than those living with a non-drinking adoptive parent, suggesting an environmental effect. However, those with alcohol-dependent biological parents displayed a range of 'disinhibited' behaviours, reflecting what the study's authors argue is the inherited

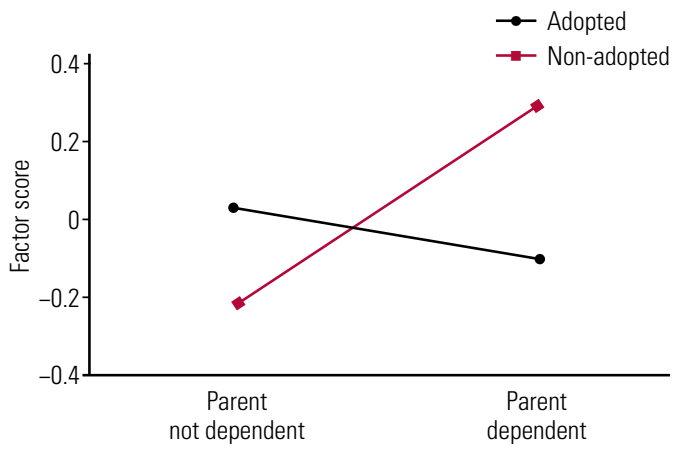

Effect of parental alcohol dependence on standardised disinhibition factor scores in adopted and non-adopted adolescent offspring (after King 2009, with permission).

disinhibited predisposition. Figure 2 shows that the disinhibition is passed to the biological child of drinking parents; the adopted child of the drinking parent is 'protected' from the disinhibition syndrome.

These datasets support the view that what is transmitted genetically is not a specific predisposition to misuse substances, but a broad behavioural disposition. This disposition is likely to include clinical syndromes such as attentiondeficit hyperactivity disorder (ADHD) and conduct disorder, each of which is independently predictive of substance misuse; these risk factors potentially act throughout adolescence (Lynam 2009; Beiderman 2010; Langley 2010). It appears also to include new forms of disinhibitory psychopathology such as cyberbullying (Sourander 2010).

\section{'Self-medication'}

Self-treatment may be another contributory factor in substance misuse. Forms of psychopathology such as bipolar disorder and post-traumatic stress disorder (PTSD) are independently associated with higher risk of substance misuse (Goldstein 2010), perhaps especially among the most vulnerable in society (Bender 2010). The effect of depression on risk of substance misuse appears substantially reciprocal, but depression may more often lead to misuse in females (Gallerani 2010). Similarly, a study of females with PTSD that included older adolescents showed that successful treatment of PTSD predicted reduction in substance use but not vice versa (Hien 2010). In a large adolescent population study, depression contributed to the uptake of smoking, and continued smoking contributed to 'a dampening or levelling off of depression symptoms'. These findings, perhaps due to the modulating effect of nicotinic acetylcholine receptors on the release of neurotransmitters, suggest that smoking is an 'effective' self-therapy for depression (Audrain-McGovern 2009). 


\section{Environment}

Environmental factors interact with predisposition. For instance, constrained environments (e.g. those characterised by strong family bonds or those in which substances are less available) appear to suppress genetic risk, whereas unconstrained or stressful environments give it freer rein. Indeed, Hicks et al (2009) concluded that the greater the environmental stress, the greater the genetic effect. In disorganised environments with easy access to substances, or in which young people are left to their own devices, predispositions are more likely to be fulfilled than in environments where access to substances is limited, a potential geneenvironment interaction. Social adversity may not so much 'cause' misuse as permit it.

\section{Pre-existing mental illness}

Swendsen et al (2010) examined risks posed by a range of mental disorders and the degree to which substance use, misuse and dependence might be eliminated through their treatment. Analysing follow-up data from the US National Comorbidity Survey, and consistent with other longitudinal studies (e.g. Copeland 2009), they argue that risk is linked to affective disorders but that preexisting behavioural disorders, including ADHD and conduct disorder, are the most powerful antecedents of substance misuse and that their successful early treatment could reduce illicit drug use and dependence by more than 70\%. Indeed, early treatment of ADHD appears to reduce risk of later misuse: in a longitudinal case-control study of girls with $\mathrm{ADHD}$, those treated with stimulants were $73 \%$ less likely to develop a substance use disorder (Wilens 2008).

\section{Intervention}

Knowledge concerning the effectiveness of intervention derives mainly from studies of prevention and treatment. However, the body of high-quality randomised controlled trial (RCT) research exclusively concerning young people is relatively limited. Consequently, in the search for useful insights and reflecting the often complex comorbid conditions of those presenting to services, it may be possible cautiously to extrapolate from treatment trials of complex adult psychopathology.

\section{Prevention programmes}

Riggs et al (2009) presented a long-term followup (from 11 to 28 years of age) of a universal intervention in the form of an early-adolescent substance misuse prevention programme. This comprised 10-13 approximately monthly teacher-led sessions of 'resistance skills' and 'social-normative change', a five-session booster 12 months later (age 13), and a parent programme including interactive sessions about homework and parent-child communication. Significant programme effects emerged at age 15 (2 years after the end of the programme) and appeared to increase until age 17, with differences between the intervention and control groups persisting thereafter. A European study has reported reduced drunkenness and less frequent cannabis use following a teacher-led classroom intervention (Faggiano 2010). A parent-only intervention was significant at 12-month outcome but a pupilonly intervention had no effect on any outcomes (Koning 2009). However, two sessions of a schoolbased targeted intervention involving group work with at-risk young people compared with no intervention yielded reduced illicit use over the 2-year follow-up (Conrod 2010). Perhaps by helping mothers develop a sense of mastery, nurse home visiting of vulnerable mothers during their pregnancy and the first 2 years of their children's lives was linked to reduced affective symptoms and substance misuse in the children 12 years later (Kitzman 2010).

These data suggest that altering school curricula to attend to wider aspects of personal development can be effective in enhancing life skills, thus reducing later problem behaviours, and that combined and booster interventions may be important for detecting sustained effects in universal interventions. However, targeted interventions have the advantage of excluding those at low risk and, because of their smaller scale, are likely to be cheaper. What the exact balance of universal and targeted programmes should be remains unclear and is likely to depend on local social and political priorities as much as on science. Prolonged follow-up can detect important delayed or sleeper effects, perhaps reflecting altered developmental trajectories. Any interventions should be evaluated in different cultures. If successful, they may be cost-effective and may benefit whole societies, although reasonable fidelity to the original concept or technology is likely to be important.

\section{Treatment}

Opportunistic brief interventions in healthcare settings have shown positive effects on adult drinking. Consistent with this, focusing on adolescents exposed to aggression and substance use, a half-hour therapy in an emergency department reduced reported exposure to violence and aggression at 3-month and alcohol consequences at 6-month follow-up compared with an information brochure control intervention (Walton 2010). 
The precise components of a successful brief intervention for young people are not known, but such a finding shows that even taking a substance history and briefly discussing its findings may be helpful, opening to a wide range of clinicians the possibility of intervening.

In an RCT targeting substance misusing adolescents with ADHD, individual psychotherapy was linked with significant reduction in substance misuse (Riggs 2011). Those additionally treated with long-acting osmotic-release methylphenidate (OROS MPH) over the course of the 4-month trial showed additional benefits. Diversion of the methylphenidate (e.g. selling it on) and interaction with drugs of misuse were not reported, allowing the authors to state that OROS MPH is safe to use in this population. Focusing on opiate-dependent adolescents, Woody et al (2008) reported the greater efficacy (as measured by abstinence) of 12 weeks as opposed to 2 weeks of buprenorphine treatment and concluded that a case exists for longer-term maintenance for this group, supplemented by psychosocial interventions.

Adolescent development occurs in 'an ecology of nested systems', such as peers, family, schools, recreation and juvenile justice. Within these systems, the relative influence of attachments to family and school versus, for instance, to deviant peers may shape behaviour (Liddle 2009). Multidimensional family therapy (MDFT) focuses on engagement of the young person and family, targeting adolescent, parental and family interactions as well as extra-familial domains of functioning (Henderson 2009).

Henderson et al (2010) conducted a secondary analysis of two RCTs comparing MDFT with either cognitive-behavioural therapy (CBT) or enhanced (through support with engagement and transport) treatment as usual. This demonstrated that, for milder forms of misuse, the more intensive MDFT treatment had no greater impact than less intensive CBT (Fig. 3). However, statistically significant differences favouring MDFT emerged among those with indicators of more severe substance misuse. Consistent with clinical experience, this important interaction seems to confirm that treatment should be tailored to the severity and complexity of the presenting problems.

Prognosis may be related to aftercare. In an 8-year follow-up of adolescents who had received in-patient treatment for alcohol or drug dependence, Kelly et al (2008) showed a relationship between abstinence and attendance at Alcoholics or Narcotics Anonymous meetings. One meeting per week independently predicted abstinence, and most attending three meetings were abstinent. Attendance during the first 6 months after discharge from hospital was associated with abstinence but this effect diminished over the period. Kaminer et al (2008) demonstrated reduced relapse rates among young people with alcohol use disorders initially treated as inpatients who were offered aftercare comprising face-to-face contact but not those offered only telephone contact, underlining the importance for many of a continued relationship with a helping other or others.

\section{Research implications}

A review of the 'first 10 years' of the US National Institute on Drug Abuse Clinical Trials Network, which mainly focuses on adult misusers, concluded that the overall efficacy of short-term detoxification was 'poor', so that 'only' $29 \%$ had opiate-free urine post-treatment (Wells 2010). The review concluded that 'the pooled impact on substance use, especially on maintenance of reduction or abstinence, of all the trials is disappointing'. It noted too the debate in the literature about the degree to which specific psychotherapeutic methods account for variance in outcome, adding that 'care should be taken in simply continuing to test one treatment after another in designs similar to those that have been employed'. The authors concluded that combinations of treatments, and flexible treatment protocols, more focus on subcomponents of treatments such as therapist effects and longer-term follow-up to detect sleeper effects are required.

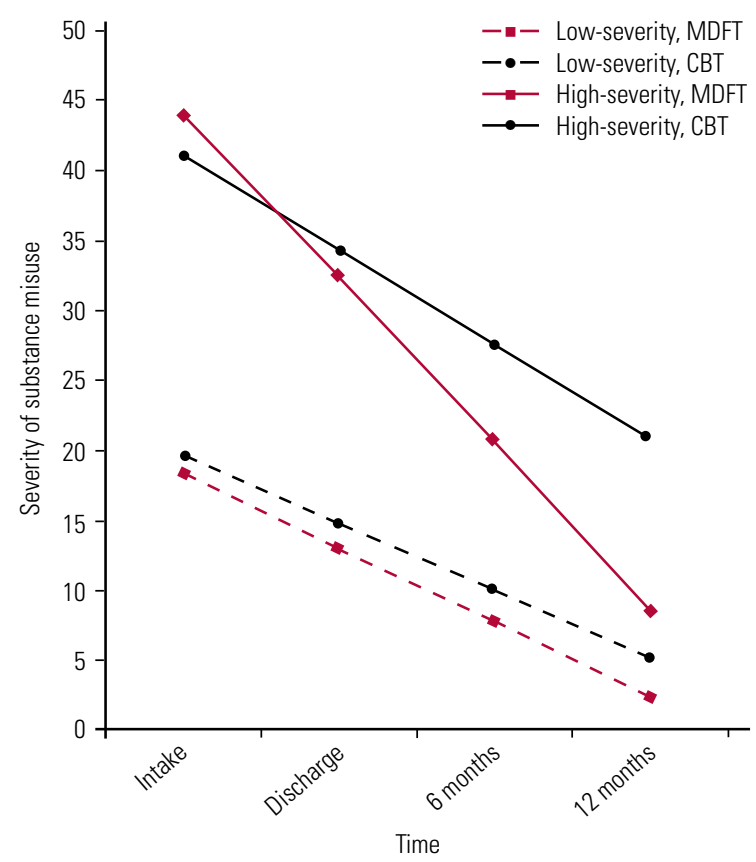

FIG 3

Treatment effect differences for milder and more severe substance misuse. MDFT, multidimensional family therapy; CBT, cognitive-behavioural therapy (after Henderson 2010, with permission). 
${ }^{\dagger}$ For a discussion in Advances of DBT, MBT and other therapies for personality disorder, see Bateman AW, Tyrer P (2004) Psychological treatment for personality disorders, 10: $378-388$. Ed.

\section{Treatment for individuals with features of personality disorder}

\section{Borderline personality disorder}

Many young substance misusers display impulsive aggression, self-harm, interpersonal difficulties, affective distress and mistrust. These are core features of borderline personality disorder, and adults exhibiting them have shown benefits from sustained structured interventions such as dialectical behaviour therapy (DBT) and mentalisation-based treatment (MBT). ${ }^{\dagger}$

In a trial involving adults with borderline personality disorder, $59 \%$ of whom reported lifetime comorbid substance use disorders, McMain et al (2009) compared DBT with expert general psychiatric management (case management, psychodynamic psychotherapy and medication). The core strategies used in the DBT involved balancing validation of patients' experience with behavioural change to help them develop more effective coping strategies, thereby eliminating behavioural dyscontrol. Over a 12-month period, 25 therapists delivered $30+$ sessions to 183 patients, $62 \%$ of whom completed treatment. Both treatments were associated with equivalent symptomatic improvement.

In another trial, Bateman \& Fonagy (2009) compared MBT with structured clinical management (crisis contact and crisis plans, pharmacotherapy, general psychiatric review, and written information about treatment). The aim of MBT, a psychodynamic treatment, was to strengthen patients' capacity to understand their own and others' mental states in attachment contexts, thus enabling them to address their difficulties with affect regulation, impulse control and interpersonal functioning. Of the 134 adult participants, 54\% reported a substance use disorder. All were offered 18 months or 140 sessions of treatment, delivered by 11 therapists, and $75 \%$ completed at least 70 sessions in the first year. The authors concluded that focusing on psychological functions related to symptoms of borderline personality disorder (e.g. difficulty in reflecting on the mental states of oneself and others) could improve a structured programme providing generic psychological support.

Oldham (2009) commented that 'often the biggest hurdle is to engage the patient in treatment - to establish a partnership that can endure so that any one of the many types of effective treatments can take hold and lead to lasting change'. He speculates that the overarching goal of therapy may be to enable rational control of emotion, to 'teach the cortex to control the amygdala'.
Overall, these studies suggest that a coherent package of interventions, sustained over at least 6 months, involving relatively intensive contact can usefully affect symptoms often considered intractable. Taken with the MDFT data, they may also suggest a dose-response effect, so that complex psychopathology requires more elaborate and sustained interventions.

\section{Antisocial personality disorder}

Adult antisocial behaviour has close links with child and adolescent conduct disorder, which are closely entwined with substance misuse. As treatment studies of adolescent conduct disorder are rare (Riggs 2011), it is instructive to consider lessons from adult treatment studies.

Frank antisocial behaviour can appear intractable. However, even among those with antisocial personality disorder, observer ratings of change in therapeutic alliance predict behaviour change (Polaschek 2010). A Cochrane review of psychological interventions for antisocial or dissocial personality disorder (Gibbon 2010) found 6 months of treatment that included contingency management to have a positive effect on attendance at counselling sessions as well as on social functioning and cocaine use (assessed by an addiction severity measure). Twenty-four weeks of CBT was associated with a significant reduction in cocaine-positive urine samples. However, CBT was not associated with change in verbal or physical aggression, compared with treatment as usual (TAU). As both interventions were associated with reduced aggression, high-quality TAU could be an effective intervention. Indeed, as the participants came from mental health and forensic services within the National Health Service (Davidson 2009), it is likely that TAU was a reasonably sustained intervention emphasising engagement, general supportive interventions and problem-solving.

\section{National guidance}

The UK National Treatment Agency (NTA) has the responsibility for 'improving the availability, capacity and effectiveness' of services for drug misusers in England. It acknowledges the need to address the complex background against which misuse often occurs. However, its website also claims that "most young people need to engage with specialist drug and alcohol interventions for a short period of time, often weeks, before continuing with further support elsewhere' (www. nta.nhs.uk/young-people.aspx). These may not be compatible aims. Indeed, NTA data reveal that, of the approximately 24000 young people aged 
16 and below seen in English substance misuse services, only $8 \%$ received family interventions (National Treatment Agency for Substance Misuse 2011). Only 3\% were 'referred on'. Despite the NTA's aspirations, most young people in the UK receive a relatively brief intervention that excludes considerations of family relationships or collaboration with other agencies. This work is likely to be short of what could be achieved.

\section{Conclusions}

The evidence base suggests that substance misusing young people, who comprise perhaps $5-10 \%$ of the adolescent population, display a constellation of behavioural and emotional difficulties. These phenomena are substantially genetically influenced so that, in general, even dysfunctional parents should not be 'blamed'. Understanding this can aid empathy and family engagement. It is possible to prevent some substance use through school-based programmes (Box 1). Existing users may show a measurable response to very brief interventions offered opportunistically. It follows that any health or social care professional encountering a young user should, in a non-judgemental way, take a substance use history and thoughtfully feed back and discuss the findings. However, especially among those below 16 , substance use may be a marker of more profound or safeguarding problems that are likely to need extensive assessment or referral. In the UK context, multifaceted interventions, the components of which are becoming apparent, require engagement and the capacity to organise a coalition of services to deliver a coherent and substantial package of intervention probably

BOX 1 Practice implications

- Relatively simple interventions targeted on emergency department attenders or at-risk pupils identified by questionnaire may reduce substance misuse and related behaviours

- In light of the complex psychopathology that accompanies substance misuse, especially more severe misuse, an effective treatment system should have the capacity to:

engage families and improve relationships with parents/carers

target the 'ecology': the young person's relationship with leisure, education, work, criminal justice system distance young people from substance-using peers be sustained over at least months offer intensive contact lasting at least some months. However, this type of response is not universal; treatment needs more closely to follow the emerging evidence base.

\section{References}

Advisory Council on the Misuse of Drugs (2010a) Consideration of the Naphthylpyrovalerone Analogues and Related Compounds. ACMD (http://www.homeoffice.gov.uk/publications/drugs/acmd1/naphyronereport?view=Binary)

Advisory Council on the Misuse of Drugs (2010b) Consideration of the Cathinones. ACMD (http://www.homeoffice.gov.uk/publications/drugs/ acmd1/acmd-cathinodes-report-2010?view=Binary).

Advisory Council on the Misuse of Drugs (2011) Consideration of the Novel Psychoactive Substances ('Legal Highs'). Home Office (http:// www.homeoffice.gov.uk/publications/agencies-public-bodies/acmd1/ acmdnps2011?view=Binary).

American Psychiatric Association (1994) Diagnostic and Statistical Manual of Mental Disorders (4th edn) (DSM-IV). APA.

American Psychiatric Association (2010) DSM-5 Development: Substance Use Disorder. APA (http://www.dsm5.org/ProposedRevisions/Pages/ proposedrevision.aspx?rid=431)

Audrain-McGovern J, Rodriguez D, Kassel J (2009) Adolescent smoking and depression evidence for self medication and peer smoking mediation. Addiction 104: 1743-56.

Bateman A, Fonagy P (2009) Randomized controlled trial of outpatient mentalization-based treatment versus structured clinical management for borderline personality disorder. American Journal of Psychiatry 166: 1355-64.

Beiderman J, Petty C, Monuteaux M, et al (2010) Adult psychiatric outcomes of girls with attention deficit hyperactivity disorder: an 11 year follow up in a longitudinal case-control study. American Journal of Psychiatry 167: 409-17.

Bender K, Ferguson K, Thompson S, et al (2010) Factors associated with trauma and posttraumatic stress disorder among homeless youth in three US cities: the importance of transience. Journal of Traumatic Stress 23: 161-8.

Collishaw S, Maughan B, Goodman R, et al (2004) Time trends in adolescent mental health. Journal of Child Psychology and Psychiatry 45: $1350-62$

Collishaw S, Maughan B, Natarajan L, et al (2010) Trends in adolescent emotional problems in England: a comparison of two national cohorts twenty years apart. Journal of Child Psychology and Psychiatry 51: 885-94.

Conrod P, Castellanos-Ryan N, Strang J (2010) Brief, personality-targeted coping skills interventions and survival as a non-drug user over a 2-year period during adolescence. Archives of General Psychiatry 67: 85-93.

Copeland W, Shanahan L, Costello E, et al (2009) Childhood and adolescent psychiatric disorders as predictors of young adult disorders. Archives of General Psychiatry 66: 764-72.

Costello EJ, Erkanli A, Copeland W, et al (2010) Association of family income supplements in adolescence with development of psychiatric and substance use disorders in adulthood among an American Indian population. JAMA 303: 1954-60

Davidson K, Tyrer P, Tata P, et al (2009) Cognitive behaviour therapy for violent men with antisocial personality disorder in the community: an exploratory randomized controlled trial. Psychological Medicine 39: 569-77.

European Monitoring Centre for Drugs and Drug Addiction (2011) Cocaine and crack cocaine: prevalence and patterns of use. In Annual Report 2011: The State of the Drugs Problem in Europe. EMCDDA (http:// www.emcdda.europa.eu/online/annual-report/2011/cocaine/3).

European Monitoring Centre for Drugs and Drugs Addiction (2012) New drugs detected in the EU at the rate of around one per week, say agencies (News Release No. 2/2012). EMCDDA (http://www.emcdda.europa.eu/ news/2012/2). 


MCO answers
$\begin{array}{lllll}1 \mathrm{c} & 2 \mathrm{~d} & 3 \mathrm{a} & 4 \mathrm{e} & 5 \mathrm{~d}\end{array}$

Faggiano F, Vigna-Taglianti F, Burkhart G, et al (2010) The effectiveness of a school-based substance abuse prevention program: 18-month followup of the EU-Dap cluster randomized controlled trial. Drug and Alcohol Dependence 108: 56-64.

Fuller E, Sanchez M (eds) (2010) Smoking, Drinking and Drug Use among Young People in England 2009. NHS Information Centre for Health and Social Care (http://www.ic.nhs.uk/pubs/sdd09fullreport).

Gallerani C, Garber J, Martin N (2010) The temporal relationship between depression and comorbid psychopathology in adolescents at varied risk for depression. Journal of Child Psychology and Psychiatry 51: 242-9.

Gibbon S, Duggan C, Stoffers J, et al (2010) Psychological interventions for antisocial personality disorder. Cochrane Database of Systematic Reviews 6: CD007668.

Goldstein B, Bukstein 0 (2010) Comorbid substance use disorders among youth with bipolar disorder: opportunities for early identification and prevention. Journal of Clinical Psychiatry 71: 348-58.

Green J, McLaughlin K, Berglund P, et al (2010) Childhood adversities and adult psychiatric disorders in the national comorbidity survey replication. Archives of General Psychiatry 67: 113-23.

Henderson C, Rowe C, Dakof G, et al (2009) Parenting practices as mediators of treatment effects in an early-intervention trial of multidimensional family therapy. American Journal of Drug and Alcohol Abuse 35: $220-6$

Henderson C, Dakof G, Greenbaum P, et al (2010) Effectiveness of multidimensional family therapy with higher severity substance-abusing adolescents: report from two randomized controlled trials. Journal of Consulting and Clinical Psychology 78: 885-97.

Hibell B, Guttormsson U, Ahlström S, et al (2012) The 2011 ESPAD Report: Substance Use among Students in 36 European Countries. Swedish Council for Information on Alcohol and other Drugs (CAN).

Hicks B, South S, DiRago A, et al (2009) Environmental adversity and increasing genetic risk for externalizing disorders. Archives of General Psychiatry 66: 640-8.

Hien D, Jiang H, Campbell A, et al (2010) Do treatment improvements in PTSD severity affect substance use outcomes? A secondary analysis from a randomized clinical trial in NIDA's Clinical Trials Network. American Journal of Psychiatry 167: 95-101.

Impinen A, Mäkelä P, Karjalainen K, et al (2010) High mortality among people suspected of drunk-driving: an 18-year register-based follow up study. Drug and Alcohol Dependence 110: 80-4.

Iversen $L$ (2009) Re: ACMD consideration of mephedrone land related cathinones) (Letter to the Home Sectretary). Advisory Council on the Misuse of Drugs (http://www.homeoffice.gov.uk/publications/drugs/ acmd1/acmdmephedrone?view=Binary).

Johnston LD, O'Malley PM, Bachman JG, et al (2009) Monitoring the Future. National Results on Adolescent Drug Use: Overview of Key Findings, 2009 (NIH Publication No. 10-7583). National Institute on Drug Abuse (http://monitoringthefuture.org/pubs/monographs/ overview2009.pdf).

Kaminer Y, Burleson J, Burke R (2008) Efficacy of outpatient aftercare for adolescents with alcohol use disorders: a randomized controlled study. Journal of the American Academy of Child and Adolescent Psychiatry 47:1405-1412.

Kelly J, Brown S, Abrantes A, et al (2008) Social recovery model: an 8-year investigation of adolescent 12-step group involvement following inpatient treatment. Alcoholism: Clinical and Experimental Research 32: 1468-78.

King S, Keyes M, Malone S, et al (2009) Parental alcohol dependence and the transmission of adolescent behavioral disinhibition: a study of adoptive and non-adoptive families. Addiction 104: 578-86.

Kitzman H, Olds D, Cole R, et al (2010) Enduring effects of prenatal and infancy home visiting by nurses on children. Archives of Pediatrics \& Adolescent Medicine 164: 412-8.

Koning I, Vollebergh W, Smit F, et al (2009) Preventing heavy alcohol use in adolescents: cluster randomized trial of a parent and student intervention offered separately and simultaneously. Addiction 104: 1669-78.
Langley K, Fowler T, Ford T, et al (2010) Adolescent clinical outcomes for young people with attention-deficit hyperactivity disorder. British Journal of Psychiatry 196: 235-40.

Liddle H, Rowe C, Dakof G, et al (2009) Multidimensional family therapy for young adolescent substance abuse: twelve-month outcomes of a randomized controlled trial. Journal of Consulting and Clinical Psychology 77: $12-25$

Lopez M, Compton W, Volkow ND (2009) Changes in cigarette and illicit drug use among US teenagers. Archives of Pediatrics \& Adolescent Medicine 163: 869-70.

Lynam D, Charnigo R, Moffitt T, et al (2009) The stability of psychopathy across adolescence. Development and Psychopathology 21: 1133-53.

McGue M, lacono WG (2008) The adolescent origins of substance use disorders. International Journal of Methods in Psychiatric Research 17 (suppl 1): S30-8.

McMain SF, Links PS, Gnam WH, et al (2009) A randomized controlled trial of dialectical behaviour therapy versus structured psychiatric management for borderline personality disorder. American Journal of Psychiatry 166: 1365-74.

McQueeny T, Schweinsburg B, Schweinsburg A, et al (2009) Altered white matter integrity in adolescent binge drinkers. Alcoholism: Clinical and Experimental Research 33: 1278-85.

National Treatment Agency for Substance Misuse (2010) Substance Misuse among Young People: The Data for 2008-09. NTA (http://www. nta.nhs.uk/uploads/nta_substance_misuse_among_yp_0809.pdf).

National Treatment Agency for Substance Misuse (2011) Substance Misuse among Young People: 2010-11. NTA (http://www.nta.nhs.uk/ uploads/yp2011commentaryfinal.pdf).

Newbury-Birch D, Walker J, Avery L, et al (2009) Impact of Alcohol Consumption on Young People: A Systematic Review of Published Reviews (Research Report DCSF-RR067). Department for Children, Schools and Families (http://www.drugsandalcohol.ie/12292/3/Review_ of_existing_reviews.pdf).

Oldham J (2009) Borderline personality disorder comes of age. American Journal of Psychiatry 166: 509-11.

Polaschek D, Ross E (2010) Do early therapeutic alliance, motivation and stages of change predict therapy change for high risk, psychopathic, violent prisoners? Criminal Behaviour and Mental Health 20: 100-11.

Riggs N, Chou C, Pentz M (2009) Preventing growth in amphetamine use: long term effects of the Midwestern Prevention Project (MPP) from early adolescence to early adulthood. Addiction 104: 1691-9.

Riggs PD, Winhusen T, Davies RD (2011) Randomized controlled trial of osmotic-release methylphenidate with cognitive-behavioral therapy in adolescents with attention-deficit/hyperactivity disorder and substance use disorders. Journal of the American Academy of Child and Adolescent Psychiatry 50: 903-14.

Sourander A, Klomek A, Iknone M, et al (2010) Psychosocial factors associated with cyber-bullying among adolescents: a population-based study. Archives of General Psychiatry 67: 720-8.

Swendsen J, Conway K, Degenhardt L, et al (2010) Mental disorders as risk factors for substance use, abuse and dependence: results from the 10-year follow up of the National Comorbidity Survey. Addiction 105: $1117-28$.

Walton M, Chermack S, Shope J, et al (2010) Effects of a brief intervention for reducing violence and alcohol misuse among adolescents: a randomized controlled trial. JAMA 304: 527-35.

Wells E, Saxon A, Calsyn D, et al (2010) Study results from the Clinical Trials Network's first 10 years: where do they lead? Journal of Substance Abuse Treatment 38 (suppl 1): S14-30.

Wilens T, Adamson J, Monuteaux M, et al (2008) Effect of prior stimulant treatment for attention-deficit/hyperactivity disorder on subsequent risk for cigarette smoking and alcohol and drug use disorders in adolescents. Archives of Pediatrics and Adolescent Medicine 162: 916-21.

Woody GE, Poole SA, Subramaniam G, et al (2008) Extended vs shortterm buprenorphine-naloxone for treatment of opioid-addicted youth. JAMA 200: 2003-11. 
MCQs

Select the single best option for each question stem

1 In substance misuse, physiological dependence in young people is:

a common among heavy drinkers

b likely to be retained as a subcategory in DSM-5

c often preceded and accompanied by conduct disorder

d less likely among those who engage in early sexual behaviour

e rarely preceded by attention-deficit hyperactivity disorder.

2 The rate of illicit drug use among young people in the UK is:

a usually calculated from household surveys

b generally increasing

c declining among high-risk groups such as school truants d often reported as the number ever having tried drugs

e higher in girls.

3 Mephedrone:

a is structurally similar to amfetamine

b remains legally available as 'plant food'

c is a by-product of 'skunk' cannabis production

d rapidly produces a dependence syndrome

$\mathrm{e}$ is used to treat opiate dependence.

4 Genetically mediated predisposition to substance misuse is:

a not usually a clinically relevant factor

b usually manifest as early depression

c prevented by early adoption

d common only among those with dependence

e often associated with adolescent risk-taking.
5 Intervention concerning adolescent substance misuse should not include:

a brief interventions

b interventions beyond 3 months' duration

c multifaceted intervention tailored to the young person's needs

d immediate-release methylphenidate

e maintenance treatment for opiate dependence. 